Voix et Images

volxetimages

\title{
Mille plateaux : topographie et typographie d'un quartier
}

\section{Ginette Michaud}

Volume 14, numéro 3 (42), printemps 1989

Gabrielle Roy

URI : https://id.erudit.org/iderudit/200800ar

DOI : https://doi.org/10.7202/200800ar

Aller au sommaire du numéro

Éditeur(s)

Université du Québec à Montréal

ISSN

0318-9201 (imprimé)

1705-933X (numérique)

Découvrir la revue

Citer cet article

Michaud, G. (1989). Mille plateaux : topographie et typographie d'un quartier.

Voix et Images, 14(3), 462-482. https://doi.org/10.7202/200800ar d'utilisation que vous pouvez consulter en ligne.

https://apropos.erudit.org/fr/usagers/politique-dutilisation/ 


\section{Mille plateaux: topographie et typographie d'un quartier*}

\section{par Ginette Michaud, Université de Montréal}

Les «mille plateaux» dont j'aimerais explorer ici quelques parcours ne renvoient pas au livre de Deleuze et Guattari, mais se font plutôt l'écho de cette campagne publicitaire désormais célèbre des marchés d'alimentation Provigo qui a récemment été exploitée avec une certaine finesse dans les médias: les téléromans de Margot, les oignons de Provigo; les singeries de l'oncle JeanPierre, la banane de Provigo; la musique de Dompierre, le ketchup de... On pensera ce qu'on voudra de ces rapprochements onomastiques, fort peu surrealistes au demeurant, mais l'une des raisons du succès de cette publicité tenait sans doute au fait qu'elle présentait, par cette série de spots très brefs d'une quinzaine de secondes, une image assez prégnante, mieux, mille petits clichés de «nous-mêmes», c'est-à-dire de notre sujet collectif, sous la figure de la belle famille québécoise des années quatre-vingt aux lendemains de l'affaissement de notre Grand Récit, pour reprendre dans un autre contexte une expression de Lyotard. Nous n'étions plus ce tout collectif et homogène, cette vaste parentèle éloignée-à-l'infini-mais-qui-tenait-si-bien-ensemble, comme à l'époque de cette autre publicité célèbre, typique des années soixante-dix celle-là, où l'on nous répétait à satiété qu'il fallait se parler, parce que, selon une curieuse logique, on était devenus assez nombreux pour s'affirmer et «s'assumer», comme on disait alors (incidemment, ce verbe «'s'assumen» traverse tel un fil rouge toutes les Chroniques du Plateau Mont-Royal ${ }^{1}$ de Michel Tremblay qui me retiendront ici). La famille Provigo des années quatre-vingt n'est donc plus si vaste et si ramifiée. Moins nucléaire qu'éclatée en capsules monoparentales, elle est devenue un petit groupe désagrégé, fragmenté en autant d'individualités juxtaposées, repliées sur elles-mêmes, séparées, s'identifiant au plus près, comme ici, à un objet de consommation, jusqu'à la réification totale, l'assimilation parfaite d'un nom propre et d'une marque de commerce. Signe des temps dits postmodernes et de la culture yuppie où plus que jamais la question du référent revient avec

* Ce texte est la version remaniée d'une communication présentée à l'occasion du colloque Lire Montréal, organisé par le Département d'études françaises et le groupe de recherche Montréal Imaginaire et qui a eu lieu le 21 octobre 1988 à l'Université de Montréal.

1 Michel Tremblay, la Grosse Femme d'à côté est enceinte, Montréal, Leméac, 1978, 329 p.; Thérèse et Pierrette à l'école des Saints-Anges, Montréal, Leméac, 1980, 368 p.; la Duchesse et le roturier, Montréal, Leméac, 1982, 390 p.; Des nouvelles d'Édouard, Montréal, Leméac, 1984, 312 p. Je renverrai directement à ces ouvrages en utilisant les sigles suivants: GF, TP, DR et NÉ. 
force et cherche à se faire passer, toute crue et toute brute, pour le réel luimême, ou pire encore, pour la forme même de l'art.

On se demandera peut-être ce que tout ceci a à voir, au juste, avec la représentation de Montréal dans la littérature québécoise récente: je dirai seulement pour l'instant que les effets de l'État-Provigo ne sont pas seulement repérables dans la publicité, mais aussi dans les signes plus subtils que nous offre la littérature, et notamment dans celle dite des best-sellers, ce genre où affleurent, plus massivement qu'ailleurs peut-être, toutes sortes d'indices textuels dont a très bien parlé Gilles Marcotte dans une conférence sur «Le temps du Matou» au printemps dernier ${ }^{2}$, et qui témoignent de ces changements, de ces variations quasi imperceptibles des «sensibilités», comme on dit. Ainsi, il ne faut peut-être pas prendre trop au pied de la lettre les déclarations d'intention de romanciers comme Michel Tremblay et Francine Noé $1^{3}$ lorsqu'ils avouent, par exemple, que leurs visées sont très simples, je voulais écrire un premier roman, dira Francine Noël à propos de Maryse, une histoire cohérente avec un début, un milieu et une fin et je voulais que ça soit gros parce que je voulais me faire plaisir 4 . On entend presque les soupirs de satisfaction ou de soulagement qui accueillent généralement ce genre de proposition: enfin, des romans avec des vrais personnages (entendre psychologiques), des histoires bien ficelées, des héros sympathiques, et pourquoi pas? heureux, avec lesquels le lecteur n'aura pas de mal à s'identifier, des lieux que l'on reconnaîtra aussi aisément qu'un chat - baptisons-le Duplessis ou Mélibée Marcotte - qui ferait le tour de son territoire. Disons que cet effet de réel, inhérent à toute entreprise réaliste - et Dieu sait à quel point celle-ci pèse lourd sur l'œuvre d'un Tremblay, par exemple, qui cherche presque désespérément à secouer l'illusion ou le leurre du «faire vrai» qui lui colle à la peau chaque fois qu'il en a l'occasion: la variation la plus récente de cette quasi-obsession a refait surface chez lui avec sa demière pièce, le Vrai Monde?: avais-je le droit d'aller puiser dans la «réalité», de violer mon entourage, ma famille pour l'art? - , disons donc que cet effet de réel n'est jamais loin de l'engouement que l'on porte à un objet tel que Montréal, ses rues, ses quartiers, ses parcs, objets dont on sait pertinemment qu'ils ne pourront jamais, précisément, être totalement imaginaires ou absorbés par la littérature. Ceci dit, la meilleure critique du statut problématique du référent en matière littéraire - et il n'est question que de cela ici -, c'est encore, fort heureusement, chez les romanciers eux-mêmes qu'on la trouve la mieux formulée. On aime le brut, le raide, le profond, le vernaculaire, le particulier, le saignant vif, déclare quelque part Marie-Lyre Flouée en résumant de façon

2 Voir Gilles Marcotte, «Le temps du Matou», conférence du cycle «Autrement, le Québec», organisé par le Département d'études françaises au printemps 1988 (à paraître dans Paragraphes, revue du Département d'études françaises au printemps 1989).

3 Francine Noël, Maryse, Montréal, VLB éditeur, 1983, 426 p.; Myriam première, Montréal, VLB éditeur, 1987, 532 p. Désormais, les références seront indiquées directement dans le texte, au moyen des sigles suivants: $\mathbf{M}$ et MP.

4 Monique Roy, «Et Maryse vint!», le Devoir, 4 février 1984, p. 17. 
percutante cette «nouvelle» esthétique qui sera aussi, à sa façon, une critique du réalisme: - Le réalisme en art, dit Benoit, c'est aussi utile qu' une patate chaude dans un dry martini. (MP, p. 71) Ce qui est en train de changer, c'est peut-être une petite scène savoureuse de Myriam première, second roman de Francine Noël, qui le suggère le mieux: dans cette scène, il est de fait simultanément question d'un gâteau en train de cuire au four et des problèmes d'écriture de Myriam, une petite fille de huit ans qui fait le brouillon de sa première lettre d'amour, vrai problème en comparaison de ceux pour lesquels Adrien Oubedon, poète national et officiel, vient consulter Elvire, son ex-muse professionnelle, recyclée muse domestique à temps plein depuis qu'elle a eu deux enfants. La scène se passe, comme il se doit, dans la cuisine et donne à peu près ceci:

Il est en train de construire "un sinueux poème new-wave», dit-il. C'est une "Ode d Maréal, ville mercantile et imaginative». «Mercantile?» demande Elvire. «Pourquoi pas?» fait Adrien en haussant le ton. «Pompe-toi pas, Adri! C'est pas une critique!» «Oké d'abord», dit Adrien, et il continue d'expliquer le fonctionnement interne de son ceuvre qui sera faite des noms de toutes les boutiques de la rue Saint-Denis et de la rue Laurier, plus quelques-unes, triées sur le volet, des rues Rachel, Ontario et Mont-Royal. C'est la la matière brute et son art de poète consiste à organiser ce magma informe, à le prendre en charge pour le renommer.

- On dirait qu'il y a quelque chose de changé dans notre sensibilité, fait remarquer Elvire en plantant un cure-dents dans son gâteau.

Le cure-dents en ressort propre et sec.

- C'est l'époque! dit Oubedon. Le beat des années 80. Tu es terriblement nostalgique, Elvi. Et granola-rétro. (MP, p. 181)

Un cure-dents propre et $\sec$, c'est le signal indubitable que le gâteau est enfin cuit, prêt à être mangé. Malheureusement, il n'existe pas d'instrument aussi précis qui nous permettrait de mesurer en toute rigueur si les textes qui se donnent ici sous la forme, essentiellement inachevée, malléable et souple, de chroniques - aussi bien dans le cas de Tremblay que dans celui de Noël sont bien arrivés, eux, à transformer leur matière brute en cuvre esthétique, s'ils ont vraiment réussi à organiser le magma informe d'où ils sont sortis et qui leur a servi de matériau premier. L'«Ode à Maréal, ville mercantile et imaginative» d'Adrien Oubedon nous intéresse ici à plus d'un titre: d'abord, parce qu'elle fait référence de façon ironique à une autre ode trop bien connue, l'«Ode au Saint-Laurent» de Gatien Lapointe qui a été l'emblème d'une certaine poésie du pays dans les années soixante.

Une parenthèse s'impose ici: il faut en effet dire un mot de la dimension politique du transfert de valeurs qui va amener les écrivains à délaisser peu à peu le thème du pays pour l'image de la ville à partir des années soixante-dix. Car si Tremblay affirme qu'il a vraiment l'impression qu'il est né pour perpétuer une rue - On ne m'a jamais entendu dire que je décrivais le Québec. J'ai 
souvent dit que je ne suis pas un pays [notons au passage cette curieuse formulation (dénégation?), de la part de quelqu'un qui dit ne pas être le porte-parole d'une collectivité], même pas une province, même pas une ville, même pas un quartier, mais une rue $e^{5}$, c'est plutôt l'impression contraire qui se fait insistante à la lecture des Chroniques du Plateau Mont-Royal ou de la fresque collective décrivant la génération des trois $\mathbf{M}$ de Montréal, Maryse-Marité-MarieLyre-Flouée (MLF pour les intimes). Car il s'agit bien pour Tremblay et pour Noël, en décrivant une rue et même une seule rue - c'est-à-dire à la limite cet escalier, ce coin de parterre clôturé et ces cris dans la ruelle 6 qui, comme le dira Raymond Montpetit, représentent avec une évidence toute particulière Montréal à nos yeux et à nos oreilles -, de décrire non seulement cette rue, mais bien le quartier, la ville, la province, le pays, et l'univers tout entier qui y seraient contenus. En ce sens, le repli sur Montréal est ambivalent: la ville est à la fois la peau de chagrin du pays rétréci, mais elle peut être aussi le lieu qui déplace et fait circuler à une tout autre échelle les questions relatives à l'identité. Je ferme, provisoirement du moins, cette parenthèse.

Mais revenons à l'«Ode à Maréal», qui est bien plus instructive encore. Passer de l'«Ode au Saint-Laurent» à celle d'Adrien Oubedon, passer du genre noble et pur de la poésie lyrique (il faudrait prononcer, comme la narratrice de Maryse, paésie) à celui, populaire et impur, des «gros» romans type best-seller, nécessairement mercantiles, a ainsi pour conséquence de faire revenir ce qui avait été refoulé par cette poésie du pays idéaliste et éthérée, c'est-à-dire, entre autres, la question des enjeux socio-économiques qui marquent tout rapport du sujet à la ville et également tout discours qui la prend pour objet. «Maréal, ville mercantile», ne produit certes pas un effet très poétique, la muse Elvire ne s'y trompe pas, elle perçoit tout de suite le couac, le hiatus irréparable entre l'argent, le pouvoir et l'imagination. («L'imagination au pouvoir» n'a-t-il d'ailleurs pas été le slogan le plus populaire de Mai 68 , celui qui s'est par la suite cassé les dents sur la réalité... du pouvoir?) Mais même si elle n'est pas très poétique, et peut-être parce qu'elle ne peut plus l'être comme avant, l'Ode d'Oubedon permet justement d'amorcer une critique efficace des sinueux poèmes new-wave et de leurs limites, et ce, comble d'ironie, au coeur d'une prose tout ce qu'il y a de plus prosaïque, mais qui ne s'interdit pas pour autant d'authentiques effets poétiques. Car, en outre, ce passage peut aussi être lu comme la version parodiée du fonctionnement interne de l'œuvre de Francine Noël elle-même, qui poursuit dans le diptyque formé par Maryse et Myriam première - bientôt trilogie avec la Maniaque d'Outremont, mais notons qu'on quitte ici le Plateau, comme chez Tremblay, pour la banlieue la mieux cotée de Montréal, Outremont -, un assez extraordinaire hymne à Montreal, ample chant qui n'est d'ailleurs pas dénué de lyrisme, mais d'un autre genre, plus cassé et heurté que celui de la poésie du pays des années soixante. Il y a donc ici toute une critique de la

5 Michel Tremblay, «Interview. Michel Tremblay: une société qui s'éveille», l'Actualité, vol. V, no 4, avril 1980, p. 17.

6 Raymond Montpetit, «Des lieux bâtis et habités: espace urbain et domicile privé», Urgences, nos 17-18, 1986, p. 77. 
rhétorique et des genres littéraires qui s'élabore à cause de l'inscription du sujet même de la ville, où il est clairement question non pas de nommer, mais de renommer, ce qui fait, en deux mots, toute la différence entre la réalité toute crue et l'art, entre la répétition du déjà-là et l'invention d'une forme qu'on ne pouvait même pas imaginer avant. Serait-ce aller trop loin que de suggérer que la littérature québécoise rattrape peut-être ainsi un certain retard esthétique? Dès le milieu du dix-neuvième siècle, la forme même de la ville avait en effet poussé Baudelaire, par exemple, à déplacer les limites génériques entre prose et poésie, et à créer un genre hybride, le poème en prose, qui n'allait cesser par la suite de gagner du terrain. C'est cette Idée de la prose (j'emprunte l'expression à un ouvrage récent du philosophe italien Giorgio Agamben) qui se retrouve en tout cas intimement liée au motif de la ville dans ces chroniques du Plateau.

Comme Tremblay, Francine Noël fait ici la preuve que, pour faire exister la ville, pour lui donner forme et substance et la rendre concrète 7 , pour l'écrire au sens fort du terme, il ne peut être suffisant de s'en tenir à une simple topographie, à une liste taxinomique des noms de rues, de boutiques, des lieux et places des quartiers, comme le prévoit Oubedon dans son poème new-wave, toujours en retard d'une révolution, mais qu'il faut, les mains pleines de fleur jusqu'au coude comme Elvire avec son gâteau, beaucoup plus encore, et même une pincée de poudre magique, pour faire lever toute cette pâte. Car une ville ce n'est pas d'abord, ce n'est pas seulement une certaine prolifération monumentale et architecturale, la configuration des plans d'urbanisme, les échanges commerciaux, la nostalgie des sites disparus sous le pic des démolisseurs: une ville, c'est d'abord et avant tout une certaine cohabitation, plus ou moins heureuse ou tendue, des langues et des discours.

Avant d'aller voir de plus près comment Michel Tremblay et Francine Noël vont respectivement s'y prendre pour écrire la ville dans leurs chroniques du Plateau, retoumons encore un instant sur la «vraie» rue Mont-Royal, sous cette enseigne éclairée de néons au-dessus du marché Provigo, qu'on peut apercevoir au hasard d'une promenade dans le quartier. Si d'aventure, vous vous rendez sur la rue Mont-Royal (mais vous devrez obligatoirement vous rendre sur place pour la voir, car cette annonce a justement pour particularité de n'être reproduite nulle part ailleurs en ville; nous touchons d'ailleurs là l'une des lois paradoxales du quartier, loi d'exclusivité qui pourrait se formuler ainsi: ce que vous trouverez ici, vous ne le trouverez pas ailleurs), vous pourrez donc tomber en arrêt devant la figure géante d'un garçon-serveur enjambant à ses pieds le Plateau Mont-Royal lui-même, reproduit tel un microcosme, comme s'il était vu sur une carte à vol d'oiseau ou sur une maquette pour architecte amateur. Le garçon, qui porte d'ailleurs plutôt la livrée des garçons d'ascenseur des films américains des

7 Tâche peut-être impossible, si le problème est, comme l'a souligné Pierre Nepveu dans sa communication au colloque Lire Montréal, que Montréal est par essence une ville trop imaginaire, jamais assez «concrète». Comme Montréal n' «existe» pas, ou à peine, il s'ensuit qu'on ne peut pas - et c'est à la fois sa limite et sa chance, son énigme particulière - vraiment la lire. 
années cinquante que celle des serveurs de Provigo, tient dans ses mains... un plateau, bien entendu, où l'on a déposé, telle une carte de visite, l'inscription mille plateaux. Représentation kitsch, plus grande que nature, à l'image de ce quartier hyperbolique, où l'on nous présente, si j'ose le jeu de mots, le sujet qui nous occupe ici sur un plateau d'argent, dans une mise en abyme un peu naïve peut-être, mais qui fait rêver, comme le signe à la fois éminemment lisible et opaque de ce que le Plateau Mont-Royal en est venu à symboliser de Montréal, petite partie métonymique devenue soudain plus grande que le tout, entité géographique à part entière presque détachée du reste, îlot dans l'île.

Car s'il est un quartier qui a reçu un traitement imaginaire royal, démultiplié selon plusieurs angles et perspectives dans la littérature québécoise des années soixante-dix et quatre-vingt, s'il est un quartier qui a été «mis sur la carte», index et échelles à l'appui, c'est bien ce quadrilatère, incertain plateau d'ailleurs, géographiquement parlant, puisque qu'il ne s'érige pas vraiment en hauteur mais, formation érodée, est étagé en contrebas de la Montagne (on y accède le plus facilement par l'artère qui le traverse de part en part, la rue Mont-Royal). Il faut essayer de circonscrire les limites de ce quadrilatère pour comprendre à quel point l'aménagement de ce territoire est totalement imaginaire: le Plateau s'étend grosso modo, selon un périmètre variable ${ }^{8}$, au sud jusqu'à la rue Sherbrooke, au nord jusqu'au boulevard Saint-Joseph (avec tendance marquée, dans les années quatre-vingt, à pousser une pointe jusqu'à Laurier et au-delà), à l'ouest jusqu'à la rue Saint-Laurent bien entendu, la rue principale, la Main avec laquelle à Montréal tout commence, à l'est jusqu'au parc Lafontaine, mais voilà sans doute le point cardinal où les bords de la carte sont les plus imprécis, quelque peu frangés. Il y a toutes sortes de Plateaux, et s'il n'y en a pas mille, il y en a tout au moins une bonne dizaine, dont les plus connus sont ceux des Chroniques de Tremblay, dont le centre est très précisément le coin des rues Fabre et Gilford ${ }^{9}$, de l'Hiver de force de Réjean Ducharme (le Plateau, ici, est plutôt déporté vers l'avenue du Parc et le parc de l'Esplanade 10), de la fameuse binerie du Matou d'Yves Beauchemin, entre autres. L'espace me manque pour faire une visite

8 Je suis consciente que le périmètre que je propose ici apparaîtra aux yeux de certains comme l'hérésie même. Je m'appuie non sur les points de repère du quartier «đéel» (mais quels seraient-ils?), mais sur ceux, singulièrement indécis et flottants, qu'on trouve dans les ceuvres en question. Si Michel Tremblay respecte de façon tout à fait cohérente le découpage paroissial dans un Plateau qui reste tout ce qu'il y a de plus villageois ('action se situe en 1942), il est tout aussi significatif que, dans l'univers de Francine Noèl, où le Plateau est devenu l'image même de la ville dans sa totalité, les frontières du quartier outrepassent régulièrement ses limites dites naturelles.

9 Robert Mane, «L'image de Montréal dans les chroniques du Plateau Mont-Royal», Études canadiennes, no 19, décembre 1985, p. 122.

10 Entre les Plateaux de Tremblay et de Noel, vient en effet s'intercaler, chronologiquement et spatialement, celui de Réjean Ducharme, avec ses marginaux, André et Nicole Ferron, pigistes en dérive, qui habitent de fait ses marges. C'est toute une étude qu'il faudrait consacrer aux rapports très particuliers de ces locataires - il faut prendre le mot à la lettre dans leur cas - qui s'installent à peine dans un lieu, quel qu'il soit, ne l'habitent pas (habite-t-on une rame de métro, par exemple?) et ne 
guidée et comparée de tous ces plateaux et je parlerai donc seulement ici des volumineuses chroniques, par ailleurs fort différentes de ton, de forme et de contenu, de Michel Tremblay et de Francine Noël, parce qu'on peut être frappé en les lisant par un certain nombre de résonances et de chassés-croisés tissant tout un réseau intertextuel entre ces deux cuvres et parce que, au-delà des retours de détails et de motifs, ces deux textes engagent l'un avec l'autre, me semble-t-il, un assez fascinant dialogue, non seulement en ce qui a trait à la question de l'espace proprement dit, c'est-à-dire de la topographie du quartier du Plateau, marquage qui constitue toujours le premier effort pour s'approprier la ville, pour fonder un lieu et l'habiter, mais peut-être surtout par le rapport très complexe au temps que ces deux chroniques cherchent également à inscrire au moyen d'une durée longue qui prend la forme, chez l'un et chez l'autre, d'une remontée dans le temps, d'un véritable espacement dans le temps. Ce n'est pas encore tout à fait la recherche d'une ville perdue, mais il y a bien quelque chose de cet ordre, un certain rapport proustien au temps, qui joue ici.

Ainsi, on sait que tout le cycle romanesque de Tremblay est venu de son thêâtre, avec des allers-retours très complexes entre ces deux tranches de son œuvre - Albertine, par exemple, ne devenant pleinement sujet thêtral dans Albertine en cinq temps, qu'après avoir acquis une véritable dimension de personnage grâce au cycle romanesque - et que Tremblay a dû, pour comprendre comment ses personnages étaient arrivés là, sur la Main, revenir à leur enfance, quelque vingt-cinq ans plus tôt ${ }^{11}$. Ce retour est aussi un retour à sa propre enfance et une extraordinaire mise en scène ${ }^{12}$ de la naissance de l'auteur, puisque le 2 mai 1942 précède de peu le jour de sa propre naissance et que la Grosse Femme, le seul personnage à ne pas avoir de nom, est sans doute enceinte de lui (son enfant, une fois né, sera lui aussi longtemps désigné comme l' enfant de la grosse femme dans les chroniques ultérieures: le prière d'insérer de la Duchesse et le roturier ne croit pas si bien dire lorsqu'il parle d'un livre à lire par-dessus l'épaule de sa mère).

cessent de marcher, mais sans but, dans la ville. Suggérons seulement pour l'instant que l'une des raisons de l'attrait exercé par l'Hiver de force (Paris, Gallimard, 1973) tenait au fait que ce roman-chronique décrivait de façon absolument précise, quasi hallucinée, comment un couple vivait sur le Plateau, ses usages, ses manies, ses habitudes, bref, le réseau de ses faits et gestes les plus banals et quotidiens: lire la ville à partir des années soixante-dix, c'est aussi essayer de rendre compte de cela, qui défie par sa platitude toute description, celle-ci s'attachant par définition au monde des choses visibles, le plus souvent des choses vues.

11 Dans l'entretien qu'il accordait à l'Actualité, Michel Tremblay ajoutait: J'ai vraiment l'impression, après avoir essayé toutes sortes d'affaires, que je suis né pour perpétuer une rue. [...] Je l'ai dit souvent et je le pense encore. C'est pour ça qu'avec la Grosse Femme d'à côté est enceinte, je suis revenu 25 ans avant les Belles-Saurs, et que j' ai tout recommencé (l'Actualité, avril 1980, p. 17).

12 Notons que 1942 n'est pas seulement dans ce contexte le fondement d'un mythe personnel dans l'œuvre de Tremblay (qui est né le 25 juin 1942), mais que cette année marque également le tricentenaire de la fondation de Montréal, ce qui donne encore plus de poids à l'hypothèse selon laquelle nous nous trouvons, avec ces chroniques du Plateau, devant la forte conjonction d'une double construction mythique et fantasmatique, individuelle et collective, dont les éléments cherchent ainsi à tout prix à se fondre l'un dans l'autre et à naître enfin ensemble, dans un même temps retrouvé. 
La question de l'enfance est également au cœur des chroniques de Francine Noèl. Dans le cas de Maryse et surtout de Myriam première avec toute cette histoire des canaux et des ruisseaux souterrains et enterrés de Montréal, c'est toute une coupe géologique de l'ancienne ville qui sera mise à découvert, en même temps que cette stratification historique du sous-sol engage également les personnages à retracer pour eux-mêmes leur propre généalogie - dans l'univers de Myriam première, les personnages circulent dans la ville, à la fois dans le Montréal fictif et réel de 1908, dans celui des années vingt et dans celui d'aujourd'hui -, généalogie qu'ils ont le fantasme, comme chez Tremblay, de vouloir toute matrilinéaire. S'il n'y a pas de pays sans grand-père, il semble bien en effet qu'il n'y ait pas davantage de ville sans grand-mère: on pense bien entendu au personnage de Victoire chez Tremblay qui règne sur toute la maisonnée et tient sous son verbe haut et dru - même après sa mort, ces célèbres «Dits» reviendront ponctuer tous les événements rapportés et structurer le récit - ses deux fils Édouard et Gabriel, sa bru la Grosse Femme et sa fille Albertine, sans parler des nombreux petits-enfants; mais l'on pense surtout aux deux délicieuses grands-mères de Francine Noęl, l'une originaire de la campagne (Alice Michaud qui vient du Bas-du-Fleuve, plus précisément de l'île Verte ${ }^{13}$ ), l'autre résolument urbaine, rompue aux usages du monde (Blanche Grand' Maison habite Outremont et a été mariée à un juge) et qui s'en tirera d'ailleurs mieux que son alter ego parce qu'elle est résolument ajustée au rythme de la ville, en proie à ses charmes et à ce qu'elle appelle ses petits ravissements. Ceuxci surgissent le plus souvent de façon inopinée, mais révélatrice, lorsqu'elle prend le métro et écoute les musiciens qui y jouent: cette descente aux enfers, ou dans le ventre de la ville qui, incidemment, possède le réseau underground le plus développé au monde, peut ainsi renverser le mythe ancien et devenir

13 Montréal-la-Rouge vs l'île Verte: il faudrait un jour dire un mot de la fortune littéraire de cette île dans la littérature québécoise récente. Chez Godbout (I'Isle au dragon), mais aussi chez Ferron et chez Victor-Lévy Beaulieu (Trois-Pistoles n'est après tout qu'à quelques kilomètres de l'île Verte), cette île située dans le Bas-Saint-Laurent, se donne comme la représentation d'une campagne idéalisée, mieux, de l'arrière-pays québécois, et incarne à elle seule les valeurs authentiques d'un temps plus ancien, aboli par la ville. Même dans Myriam première, et malgré l'humour tendre avec lequel Myriam accueille les propos de sa grand-mère sur son île natale, on sent poindre une certaine nostalgie: $A ̀$ l'île Verte, l' air est plus pur qu' ailleurs, le ciel est d' un bleu indélébile, et les graseilliers sont lourds de fruits verts et cependant mûrs. Là-bas, tout est mieux, toujours, C'est le pays de l'enfance d'Alice, une époque dont le souvenir s'estompe chaque jour et la fuit. [...] Elle aimerait que le jour où Alice l'amènera à l' île soit un vrai jour et pas seulement une façon de parler. (MP, p. 14) La croyance, pleine de doute déjà, de la petite fille en la parole de sa grand-mère, l'entrelacement de la mémoire et du lieu, du vrai et du faux, sont ici suggérés avec beaucoup de finesse. Par ailleurs, la Gaspésie natale de la Grosse Femme revient constamment la hanter, la submergeant de regrets, berçant ses rêves (mais il s'agit alors des mers du Sud): ici aussi, le deuil de la mère et des origines semble infini. L'île rouge - sur l'ancienne carte de Montréal que possède Laurent dans Myriam première, l'île rouge est entourée d' un gros fleuve vert (MP, p. 228) -, polluée et étouffante, s'oppose en tout cas à la verte, aérée et écologique, et il y aurait ici toute une représentation des rapports ville/pays qui mériterait d'être explorée dans une cartographie plus détaillée. 
soudain lieu euphorique. Par ailleurs, on remarquera que ces moments magiques qui ont souvent lieu dans le métro ou dans les couloirs souterrains de la ville offrent l'image même des plateaux qui m'intéresse ici: opérant de petites transgressions du code réaliste et de la linéarité narrative, ils écrasent les temps et les espaces, les catapultent sur des plans et perspectives différents. La phrase clé est ici celle des bandes dessinées: Pendant ce temps, trente pieds plus haut...

Mais revenons à cette question essentielle des perspectives et des points de vue mis en scène par ces deux œuvres, d'une part, et de la coïncidence temporelle qui jouent entre elles, d'autre part. On connaît l'argument qui sert de trame de fond au premier tome des Chroniques de Tremblay: la Grosse Femme d'à côté est enceinte raconte les menus événements qui surviennent en une seule journée, un certain 2 mai 1942 (c'est un samedi, jour de congé, où le temps, ralenti et plus intense, se dilate), convention proprement theâtrale qui facilite la présentation et la circulation des quelque vingt-deux personnages qui habitent la rue Fabre. Myriam première de Francine Noel reprend pour sa part la chronique des années floues (pour emprunter au titre d'un film récent de Sylvie Groulx), chronique qu'elle avait menée dans Maryse jusqu'en 1975, et qu'elle projette dans Myriam première huit ans plus tard, soit en 1983: le roman baigne lui aussi dans une atmosphère de fête (c'est l'anniversaire de Gabriel, le fils de Marité), par une belle journée de printemps, au tout début du mois de mai. De la rue Fabre à la rue Mentana, de 1942 à 1983, d'un mois de mai à l'autre, d'une génération à l'autre également, on peut être tenté de se demander ce qui s'est passé en quarante ans sur le Plateau, de voir comment le quartier s'est transformé, comment la perception de la ville, du balcon de la Grosse Femme au jardin bien-nommé Babylone de Marité, s'est modifiée en profondeur.

Car de l'univers de la Grosse Femme à celui de Maryse qui habite pourtant le même quartier, à quelques rues l'une de l'autre, force est de constater que nous nous trouvons ici dans deux mondes parallèles qui ont bien peu de chance de se rencontrer et sont peut-être même destinés à s'exclure. Même si on n'arrête pas dans les romans de Noel de dire comme le monde est petit, surtout dans la région du Plateau Mont-Royal (MP, p. 83) et d'en faire l'expérience, même s'il y a chez les deux romanciers une disposition de l'imaginaire fondamentalement analogue qui consiste à décrire le Plateau par un savant mélange de réalisme et de fantastique - je pense, par exemple, à la maison invisible des trois Parques dans la Grosse Femme que seuls les enfants et les fous peuvent voir, au Génie de la langue française ou au bar du Diable Vert dans Myriam première qui existe, là encore, seulement pour les enfants, les marginaux comme la punkette Marie-Belzébuth ou les écrivains en état de grâce -, on reste en effet ici devant des Montréal parallèles qui ne se croisent pas ou rarement, sauf en quelques zones d'intersection communes ${ }^{14}$. Si, de Tremblay à Noël, les quartiers

14 Les univers de Tremblay sont de fait assez étanches pour ce qui est de leurs personnages respectifs, qui ont bien peu de chances de se croiser. Cependant, il y a bien dans Maryse une zone où les femmes du Plateau de Tremblay, la génération «native» du quartier, pourrait-on dire, entrent en contact avec celles de la nouvelle 
changent vite comme à New York (MP, p. 138), on pourrait aller jusqu'à dire que ces univers romanesques donnent chacun une représentation de Montréal et du Plateau qui demeure littéralement suspendue, tels ces fameux jardins de Babylone qui étaient précisément aménagés selon de savants nivellements de terrain, de subtiles différences de niveaux et de plans, sur des plateaux différents.

De fait, lorsqu'on retrouve Maryse et ses amis au jardin de la maison sise sur la rue Mentana, on ne peut s'empêcher de penser que cette accession au jardin est également le signe de l'accession à la propriété, et qu'il s'est passé sur le Plateau ce qui s'est passé un peu partout dans les villes industrialisées en Amérique du Nord et ailleurs, c'est-à-dire la lente mais sûre gentrification d'un quartier populaire. Car ce n'est peut-être pas tout à fait la même chose d'observer la vie du quartier en immuable et immobile locataire, comme le fait la Grosse Femme du haut de son balcon, que de le faire en propriétaire, même modeste, du fond de son jardin. De Tremblay à Noêl, on voit çà et là surgir certaines retombées de ce phénomène social, de cet embourgeoisement par rénovation, notamment dans la perception et l'aménagement des lieux intérieurs, de ces vastes appartements du Plateau que l'on voit changer en même temps que leurs locataires au cours de ces chroniques. Dans le roman de Tremblay, l'appartement de la rue Fabre n'est pas du tout misérable, comme on aurait pu s'y attendre, il est encore relativement neuf en 1942 et propre, et il est grand (il s'agit probablement d'un appartement de six pièces et demie); s'il paraît petit, c'est seulement parce que trois générations et deux familles complètes s'y entassent et doivent, pour dormir par exemple, aligner quotidiennement les lits de camp dans l'étroit et sombre corridor qui traverse d'avant en arrière, de façon si caractéristique, ces appartements, de l'unique trouée lumineuse du salon double qui donne sur la rue jusqu'à la cuisine, où la remise, la shed coupe presque toute la lumière. Dans Maryse, les logements - tout particulièrement la piaule où elle et ses colocataires se chauffent en allumant ces fameux grille-pain à rabats que tous les étudiants connaissent très bien pour en avoir fait le même usage un jour ou l'autre - se sont beaucoup détériorés, ils sont peu entretenus et la population étudiante qui les occupe et qui a envahi le quartier avec la fondation de l'UQAM en 1969, n'a pas encore les moyens de les retaper. Il faudra encore attendre quelques années pour que tout le monde se mette au décapage, la spéculation allant alors bon train dans le quartier. Mais c'est peut-être la grand-mère Alice (elle est d'une autre génération, elle se rapproche d'ailleurs ici du point de vue nostalgique de la Grosse Femme) qui, parce qu'elle n'arrive pas vraiment à se transplanter de sa Gaspésie mythique à Rosemont, exprime le mieux la difficulté et la contradiction urbaine de nature quasi ontologique qui consiste à vouloir

génération représentée par Maryse et ses amies: il s'agit de la rencontre, qui se passe plutôt mal, entre Maryse et sa femme de ménage, Rose Tremblée (notons que, même orthographié différemment, c'est le nom de la mère de Maryse qui revient ici et entraîne ses résistances). Dans Myriam première, les femmes du Plateau façon Michel Tremblay feront également une apparition remarquée, sous la figure du public-cible idéal, celui qui ne se trompe jamais, qui vient assister à la première toute privée de la pièce de Maryse. 
habiter un logement, c'est-à-dire à prendre lieu et racine dans ce qui ne saurait en rien correspondre à l'idée qu'elle se fait d'une vraie maison:

Dans chacun des logements qu'elle a occupés a Rosemont, c'est la maison de sa mère qu'Alice a essayé de retrouver. Mais on ne peut pas reconstituer, pense-t-elle, un univers de deux étages dans un six et demi qui s'étend sur un seul étage. Cela manquait d'envergure, de hauteur, de profondeur. En ville, les toits sont plats et les caves souvent inaccessibles. (MP, p. 215)

Le deuil non surmonté de la maison natale, cette maison de la mère à jamais perdue en ville (dans le roman québécois de la période agriculturiste, on parle très souvent de Montréal comme d'une ville perdue, d'un lieu fait pour se perdre), ces résistances encore quelque peu bachelardiennes devant l'aplatissement littéral de l'espace urbain qui se fait jour ici auront sans doute totalement disparu à la prochaine génération, mais rien n'est sûr en ce domaine: notons que la maison de Marité, sur la rue Mentana, souscrit elle aussi aux deux étages réglementaires pour avoir droit au titre de vraie maison. A Home, not a house, disent les Anglais.

Cette première constatation sur les mille manières d'habiter un quartier à peine esquissée, et même s'il y aurait encore beaucoup à dire sur cette topographie des intérieurs et de l'intimité qui compte pour beaucoup dans l'imaginaire propre au quartier ${ }^{15}$, essayons maintenant de voir très brièvement comment ces différences relatives au statut social, à la condition économique, aux classes qui séparent les univers de Tremblay et de Noell, peuvent colorer la perception subjective que les personnages ont du quartier qui les entoure. Ainsi, pour aller tout de suite à l'essentiel, on pourrait dire que l'immobilité quasi totale de la Grosse Femme, qui ne se déplacera dans toute sa journée qu'une seule fois, de son fauteuil près de la fenêtre au balcon, lui assurera paradoxalement un point de vue beaucoup plus englobant et infaillible sur les êtres et les choses, que celui que peuvent avoir tous les autres personnages du roman qui gravitent autour d'elle. On sent en effet qu'elle tient sur eux le point de vérité, qu'elle est presque objective dans son détachement et sa neutralité bienveillante, tout

15 Disons tout de même un mot de la perception des espaces extérieurs de ce quartier qui, avec ses escaliers et ses multiples portes donnant sur leur balcon, est tout à fait typique des logements populaires montréalais érigés dans les années 1910-1930. Comme le fait remarquer Raymond Montpetit, la question de la porte d'entrée est majeure: la porte, lieu frontière du dedans et du dehors, lieu de passage du public au privé constitue le critère minimal de l'intériorité privée, traduite en termes architecturaux: Il faut autant que possible, [écrit l'abbé E. Gouin], à chaque logement habité par une famille, son escalier, sa porte sur le dehors car, note un autre observateur réformiste, une entrée spéciale pour chaque locataire [donne] à chacun l'illusion du chez-soi individuel (art. cité, p. 82). Quant aux escaliers extérieurs, longtemps décriés comme étant laids et casse-cou dans un pays où il neige, sortes d'échafaudages devenus permanents, ils sont presque devenus le symbole de la ville de Montréal, le ministère des Affaires culturelles et la Communauté urbaine de Montréal ayant même conçu une affiche qui les donne comme la contribution de la ville à l'histoire de l'architecture (Raymond Montpetit, loc. cit., p. 101). 
simplement parce qu'elle voit mieux et conserve sur la rue Fabre un regard panoptique et surplombant, le point de vue qui serait par excellence celui des narrateurs omniscients et totalisants des romans de Balzac, par exemple, écrivain-modèle qu'elle lit et affectionne tout particulièrement (de fait, il y aurait beaucoup à dire sur les rapports qu'entretient Tremblay à son «modèle», notamment en matière d'esthétique réaliste). D'autre part, dans l'univers beaucoup plus fragmenté de Maryse, où le rythme de la vie et la narration sont beaucoup plus syncopés, où tout et tout le monde est en constant mouvement (c'est la version romanesque de la théorie de la relativité d'Einstein), où les déplacements dans la ville et hors d'elle (on part beaucoup dans les romans de Francine Noël: pour le Maine, pour Paris, pour l'Amérique du Sud) sont multipliés au point de devenir la règle ordinaire, il n'y a plus de point de vue privilégié qui s'élèverait, surtout dans Myriam première, au-dessus des voix polyphoniques et toutes (relativement) égales, mais seulement des regards que l'on pourrait qualifier de rampants, parce qu'ils sont toujours prêts à se perdre au sol, au gré de circuits ou de marches dont les points de raccord, de connexion ou de disjonction, ne sont plus évidents à l'œil nu et miment plutôt l'enchevêtrement des racines et des sources souterraines de Babylone, les rhizomes des rues et ruelles transversales de Montréal, bref, il se produit ici un balaiement des. surfaces où le regard n'est plus jeté de haut, jouant de la profondeur et de la verticalité, mais vient plutôt du dessous des choses et affleure en surface - ce qui ne veut pas dire pour autant qu'il soit superficiel -, n'hésitant pas à se noyer à l'occasion dans une certaine perception confuse ou brumeuse (et n'oublions pas que Maryse est myope).

De fait, les contours du quartier se sont beaucoup rétrécis 16 dans Myriam première, autour du jardin qui est le centre de l'univers - ce qui est tout naturel pour une petite fille de huit ans (rappelons ici la figure de Tinamer de Portanqueu dans l'Amélanchier de Ferron) -, en même temps que ses frontières extérieures sont devenues plus poreuses pour les adultes. Le quartier ne protège plus aussi efficacement contre les agressions, la violence, la laideur du dehors. Cela est particulièrement clair lorsque Maryse se retrouve brusquement en zone frontalière et que le conflit des codes se fait beaucoup plus abrupt:

16 De Maryse à Myriam première, l'espace géopolitique de la ville s'est en effet considérablement resserré, la fresque a pris des proportions plus économiques, les rapports entre les différents réseaux sociaux et leurs discours se sont réduits à quelques bulles familiales et à leurs satellites amis. Cette situation narrative est sans doute liée au retrait du politique qui marque au Québec, référendum aidant, le début des années quatre-vingt, mais elle est peut-être aussi le signe d'un changement plus profond où, à l'esprit de groupe et à la grégarité générale, succède une intériorisation, opérant sur un autre mode, de la question politique. On aurait tort de ne lire ici qu'un repli ou un simple retour à l'individualisme triomphant: comme le dira Edouard, $J$ 'ai toujours vécu en troupeau, pis me v'là tout seul comme une grosse lune au milieu de rien! (NE, p. 71) Dans cet aveu s'exprime aussi la condition nécessaire pour accéder à l'œuvre d'art véritable, le passage du roman collectiviste de la tribu ou du clan au personnage non pas individuel, mais de plus en plus singulier qui s'en libère. 
Maryse ramasse sa serviette et part en courant. Elle court jusqu'au coin des rues Ontario et Sanguinet où le souffle lui manque. Elle réalise alors qu' elle est en train de remonter chez elle d pied, dans le désordre et la déroute les plus complets. Elle est mortifiée de ne pas être intervenue, tantôt. Mais ses seules armes sont rhétoriques et, dans une telle situation où le cul l'emporte sur la tête et le poing sur le coeur, elle ne pèse pas lourd: elle vaut à peine ses cent dix livres de chair d' intellectuelle. Elle marche maintenant d' un pas de fuite. Pour la première fois de sa vie, elle trouve la ville laide et voudrait aller vivre ailleurs. Le quartier, qu'elle connaît depuis son enfance et qu'elle aime bien, lui semble lamentable. Les maisons ont été démolies les unes après les autres et remplacées par des stationnements, des édifices bon marché ou des terrains vagues. Au fil des ans, la ville s'est effritée. Pendant ce travail de lente corrosion, pour s'en défendre, elle n'a pas vraiment regardé. Plusieurs Montréalais ont fait comme elle, s'exerçant à découper le réel et à imaginer, à partir d' un fragment épargné, d' une porte miraculeusement repeinte ou d' un pan de mur encore debout, comment c'était, autrefois. Ils se retrouvent maintenant dans des parkings sordides, à évoquer des splendeurs passées. Montréal n'est plus une ville mais un souvenir que se partagent quelques-uns avec un vague sentiment de nostalgie coupable. Elle traverse la rue Saint-Denis dont les magasins et les bars ont été retapés. La réfection s'arrête souvent au rez-de-chaussée et, quand on lève les yeux, on voit que les corniches des édifices sont brisées et que le mortier des pierres s'est effrité. Personne ne l'a jamais refait. Laurent prétend que le coeur de Montréal est en train de pourrir. Il dit cela sans aucune agressivité mais il a raison; il $n^{\prime}$ a pas, face à la ville, la tolérance de ceux qui y sont nés. Aujourd' hui, Maryse voit les choses comme lui, comme elles sont: laides - ou en train de le devenir - et violentes. Car la laideur est une forme de violence: entre l'érosion de la ville et le coup de poing infligé a la prostituée Barbara, il n'y a pas tellement de différence, c'est de la même chose qu' il s' agit: incurie et violence urbaine. (MP, p. 75-76)

Ainsi, la rue Boisbriand, rue du thêâtre et de la prostitution ${ }^{17}$, lieu du jeu et de la perte des identités, est-elle ce lieu ambivalent où l'on trouve le côté cour de ce petit monde et où l'on passe de l'autre côté du miroir; avec le thêatre qui crée une petite enclave encore partiellement protégée parce qu'elle est un lieu où l'art circule, cette rue laisse aussi vraiment voir l'envers du décor, les coulisses de la ville, en soulignant l'écart qui existe entre la réalité transposée et esthétisée du thêâtre et le réel brut - et brutal — de la rue, où Barbara la putain se fait tabasser par son pimp, sans que Maryse n'ait les moyens d'intervenir.

17 Le théâtre joue un grand rôle, aussi bien dans la chronique de Tremblay que dans celle de Noèl: ce théâtre dans le roman est souvent l'occasion de mises en abyme, comme on peut s'y attendre, mais aussi d'une projection sur une autre scène, en même temps qu'y joue également une importante délimitation des frontières entre genres. 
Si l'on essayait de reformuler encore autrement cette différence des points de vue dans ces deux chroniques, on pourrait suggérer, au risque toutefois de simplifier à outrance le foisonnement de ces œuvres, que les Chroniques du Plateau Mont-Royal de Tremblay semblent davantage marquées par un certain effort d'élévation, de sortie hors du périmètre du quartier selon l'axe de la hauteur, et il faut remarquer que ce périmètre, cette enceinte quasi sacrée ${ }^{18}$ qui assimile presque alors le quartier au ghetto, est de façon générale beaucoup plus précisément tracé ici que dans la chronique de Francine Noêl. Sont témoins de cette élévation certaines grandes scènes ou morceaux de bravoure qui se présentent comme le finale obligé des chroniques, le point d'orgue de longs et parfois laborieux préparatifs narratifs, comme la procession-parade de la FêteDieu dans Thérèse et Pierrette à l'école des Saints-Anges, où tous les regards sont tournés vers le pitoyable ange suspendu dans les airs (en anglais, le titre accentue d'ailleurs ce qui s'expose ainsi: Thérèse and Pierrette and the Little Hanging Angel). Pensons encore à Gérard Bleau qui, tel un nouvel Icare, grimpe tout en haut du pont Jacques-Cartier lorsqu'il songe à se suicider et rêve d'un spectaculaire saut de l'ange:

Il pensait à se jeter en bas du pont, il s'imaginait tomber en battant des bras comme pour s'envoler, il ressentait le vertige puis la joie de se savoir parfaitement libre de ses mouvements pendant cinq ou dix secondes; il jouissait de peur entre le pont et l' eau, il saluait la ville en pointant les pieds vers les rochers, il envoyait des baisers a la tour de l'île Sainte-Hélène, il faisait un bras d'honneur d̀ l'est de Montréal qui l'avait vu naître et qui lui tournait le dos pendant qu'il se sacrifiait [...] Le soleil avait déjà commencé sa longue descente vers le mont Royal lorsque Gérard revint à pas traînant vers la ville [...] (TP, p. 232-233)

Ou rappelons encore cette autre scène célèbre de la Duchesse et le roturier où un tramway ramène du théâtre, en pleine tempête de neige, les gens du quartier dans leur enceinte familière et a toutes les peines du monde à gravir la côte de la rue Sherbrooke, la ville étant quasiment toute paralysée ${ }^{19}$. Il faudrait pouvoir suivre ici en détail le parcours du tram, du Thêâtre national de la rue Papineau où la grosse machine verte et jaune avançait par d-coups en crachant ses étincelles, bateau lumineux qui traverse une mer blanche immobile en la

18 Retraçant lui-même les limites de son Montréal imaginaire, Michel Tremblay dit de la rue Fabre qu'À cette époque-là [...], Montréal était divisé en quartiers beaucoup plus que maintenant. Comme un quadrillé. J'ai jamais su qu'il y avait des Anglais dans mon bout, alors que Maureen Forrester vivait dans la même rue que moi, plus haut que Gilford. [...] Je sortais très peu du milieu francophone. Pour moi, Montréal ça finissait rue Saint-Denis. («Un parc, quelques rues et deux quartiers», le Devoir, 14 novembre 1987, p. D1, D3). Il évoque également dans cet entretien la ruelle derrière la rue Gilford qui menait jusqu' au bout du monde, le parc Laurier (la punition) et la parc Lafontaine (la récompense), quelques incursions vers l'ouest, la Main imaginée ( $J$ 'ai puisé dans ma mémoire collective personnelle)...

19 James Joyce parlait aussi de Dublin comme d'une ville paralysée et voulait laisser deviner l'âme de cette hémiplégie ou paralysie que beaucoup prennent pour une ville (Lettre à Constantin P. Curran, 1904). 
fendant en deux, laissant derrière lui une longue blessure figée, définitive, qui jamais ne se refermera (DR, p. 93), avec l'arrêt au coin de la rue SainteCatherine puis de la rue Ontario où le conducteur dut descendre trois fois de sa machine pour aller replacer son trolley qui avait débarqué du fil qui l' alimentait (la promenade prend presque des allures de chemin de croix, avec les trois chutes et le Golgotha à gravir), jusqu'au parc Lafontaine (Le restant va t'être facile à faire, c'est plat comme le creux de ma main, DR, p. 97), symbole du Plateau et image du paradis, pour Édouard qui le voit

scintiller faiblement sous le ciel noir. [...] Il regardait défiler ce vaste espace bleu et blanc que les arbres décharnés semblaient avoir défoncé tout récemment, levant vers les étoiles les paquets de neige qui s'étaient accrochés a leurs branches et qu'ils offraient dans une totale immobilité à des dieux inconnus. [...] Des larmes dont il ne compre nait pas le sens lui montaient aux yeux d la vue de cette neige parfaitement vierge [...]. (DR, p. 97)

Le lecteur comprend lui aussi, avec cette extase mystique, qu'il a devant les yeux l'une des visions renvoyant aux mystères les plus sacrés et archaiqques de Montréal. Comme l'écrit Walter Benjamin à propos de Moscou dans ses «Paysages urbains» - et que d'affinités entre Montréal et Moscou sous ce rapport -,

[...] pour connaître la ville, [il faut] la connaître sous la neige. Car chaque région doit être visitée durant la saison qui voit l'extrême de son climat. C'est à lui en effet qu'elle est adaptée et c'est seulement par cette adaptation qu'on la comprend. La vie à Moscou en hiver est plus riche d'une dimension. L'espace littéralement se modifie selon qu'il est brûlant ou glacé. On vit dans la rue comme dans une Galerie des glaces prise par le gel, chaque arrêt et chaque réflexion deviennent incroyablement difficiles. Il faut déjà une demi-journée de réflexion pour mettre dans la boîte aux lettres une lettre qui porte déja son adresse et, malgré la rigueur du froid, c'est un exploit de la volonté que d' entrer dans un magasin pour y acheter quelque chose 20 .

Cette lenteur, physique et psychique, cette pétrification de la ville sous la neige, constituent bien également ici une manière de régression du paysage urbain vers une campagne qui, même dans une métropole comme Montréal, reste seulement à moitié disparue, toujours prête à ressurgir ${ }^{21} \ldots$

20 Walter Benjamin, «Paysages urbains», dans Sens unique précédé de Une enfance berlinoise, traduit de l'allemand par Jean Lacoste, Paris, Maurice Nadeau, 1988, p. 278.

21 Benjamin dira également des rues de Moscou qu'elles sont d' une nature particulière : le village russe y joue à cache-cache. (...] Une grande ferme ou un village s'ouvre là, large et spacieux, le sol est inégal, les enfants circulent en traîneau, des remises pour le bois et les outils donnent à la façade des maisons qui, vues de la rue, ont une allure citadine, l' apparence d' une ferme russe. Des églises se dressent souvent dans ces cours, exactement comme sur la large place d'un village. La rue prend ainsi la dimension de la campagne (ibid., p. 272). Plus loin, il ajoute: Nulle part Moscou ne semble être la ville elle-même; tout au plus sa banlieue. Sans replier cette description 
Pour sa part, la chronique de Francine Noël multiplie les circuits de ses personnages, moins à partir de quelques rues bien ordonnées en abscisses et coupées à angle droit, que de lignes transversales et de ruelles, de ruisseaux afférents. Personnages et lecteurs sont ici pris dans un mouvement d'ensemble qui les entrâne dans de multiples directions, mais ils ne sont pas pour autant complètement désorientés... Pensons, par exemple, à la maison de Myriam, aussi bien partagée que celle de Tinamer dans l'Amélanchier, entre le bon et le mauvais côté des choses, avec son côté jardin - mais attention, c'est Babylone, le lieu de toutes les confusions - et son côté "courre», qui va jusqu'à la rue Boisbriand dont j'ai déjà parlé. Pensons aussi au roman des apprentissages de Maryse, véritable Bildungsroman qui prend pour modèle Elisa Doolittle et avant cette version moderne, le mythe de Pygmalion lui-même. Maryse apprendra en effet qui elle est ${ }^{22}$ en se déplaçant très littéralement et en se frottant à divers langages à la faveur de ses nombreux déménagements, trajet presque circulaire qui la ramènera tout près de son point de départ, de la très pauvre maison familiale de ses parents sur la rue Hôtel-de-Ville, à la première piaule partagée avec des copains rue Sainte-Famille où elle formera trio, sinon trinité, avec Ti-Cul Galipo et François Ladouceur, plus tard remplacé par Coco Ménard, sorte d' ange asexué, puis à l'appartement pas très beau mais lumineux qu'elle partagera ensuite avec Michel Paradis quelque part dans Outremont en-bas avant de revenir se fixer, une fois la rupture consommée, dans un appartement au troisième étage, très haut dans les arbres, plus petit, moins cher, et tout près du parc Lafontaine.

La topographie du quartier n'étant jamais plus claire qu'au moment où on en franchit les limites, je voudrais maintenant pour finir retourner sur la rue

sur celle de Montréal, il y aurait sans doute des correspondances étonnantes à établir entre ces deux villes nordiques: on lira, pour s'en convaincre, la belle description que Benjamin fait d'une promenade en traîneau: $P$ as de vue plongeante: un frôlement tendre, rapide, le long des pierres, des hommes et des chevaux. On se sent comme un enfant qui glisse sur sa petite chaise à travers l'appartement (ibid., p. 255).

22 N'oublions pas que Maryse est née d'un père irlandais, Tom O'Sullivan, et d'une mère québécoise, nommée comme il se doit Tremblay. Francine Noêl dira lors d'une entrevue que l'Irlande, ça été ma réaction très émotive au référendum. J'ai voté oui, ayant fait un cheminement analogue à celui de bien des Québécois, d'autres, apparemment, avaient fait une autre réflexion. À ce moment-là, Maryse ne pouvait plus être uniquement Québécoise. J'ai voulu une ethnie qui, par certains cốtés, ressemblerait aux Québécois. Sur les rapports de 1'Irlande, du Québec et de la stérilité, voir Maryse: Elle n' aurait pas plus de descendance qu' elle n' avait eu droit à des ascendants auxquels s'identifier: la famille n'était pas de son ressort. Elle ne tenait, ne voulait tenir, ni de sa mère francophone et bornée, ni de son père irlandais, idiot et inconsistant... Elle était issue de toutes ces bâtardises, de ces deux sous-cultures appauvries. Elle était sans racines et ne devait pas s'attarder à la question des origines [...] (M, p. 398). Dans Myriam première, Francine Noël admet cette fois avoir transféré sur la langue espagnole son rapport compliqué avec la langue anglaise (Monique Roy, «Francine Noël de Myriam première: «J'ai voulu montrer l'importance de la transmission d'une culture», la Presse, 27 février 1988, p. J-1). 
principale de notre ville-encore-un-peu-village, je veux parler de la Main, bien entendu. Il y a dans la Grosse Femme et dans Maryse deux scènes qui se répondent et qui disent de façon plus éloquente encore ce qui a changé dans l'écriture du Plateau, la seconde scène étant peut-être à sa manière la reprise et la réćcriture de la première. Il s'agit de cette scène de la Grosse femme où les femmes du Plateau prennent le tramway 52 et passent par la rue Saint-Laurent pour la plus longue ride en ville:

Mercedes avait rencontré Béatrice dans le tramway 52 qui partait du petit terminus au coin de Mont-Royal et Fullum pour descendre jusqu'd Atwater et Sainte-Catherine, en passant par la rue SaintLaurent. C'était la plus longue ride en ville et les ménagères du Plateau Mont-Royal en profitaient largement. Elles partaient en groupe, le vendredi ou le samedi, bruyantes, rieuses [...]. Tant que le tramway longeait la rue Mont-Royal, elles étaient chez elles, elles faisaient tous les temps [...]. Mais quand le tramway tournait dans la rue Saint-Laurent vers le sud, elles se calmaient d' un coup et se renfonçaient dans leurs bancs de paille tressée: toutes, sans exception, elles devaient de l'argent aux Juifs de la rue Saint-Laurent, surtout aux marchands de meubles et de vêtements, et le long chemin qui séparait la rue Mont-Royal de la rue Sainte-Catherine était pour elles très délicat à parcourir. [...] Elles n'osaient même pas regarder à l'extérieur, se disant qu'une tête vue de profil est plus difficile à reconnaître qu' une tête vue de face. Quelques-unes, mais vraiment juste quelques-unes, en profitaient même pour sortir leur chapelet. À peine faisaient-elles semblant de se boucher le nez quand une vieille Juive montait dans le tramway chargée de sacs à provisions d'où $s^{\prime}$ échappaient des queues de carottes ou de poireaux. «A' s'en va faire sa salade de pissenlits!» Elles pouffaient. «C'est-tu l'ail qu'al'a mangé hier ou ben donc son linge qu'a va laver le mois porchain qui sent fort de même?» Elles pinçaient les lèvres. «Y paraît qu'y gardent leurs transferts pour se faire du papier de toilette! [...] Mais aussitôt le coin des rues Saint-Laurent et Sainte-Catherine tourné vers l'ouest, la liesse reprenait de plus belle et emplissait le tramway de cris sonores et de rires pleins et sentis. «Vous avez failli me faire mourir, avec vos foleries, vous! « «ous étiez rouge comme une banane, $j$ 'veux dire comme une pivoine, madame Jodoin!» «D'un coup qu'a comprend le français, la Juive!» $« A$ ' comprend pas le français, al' a rien a nous vendre!» La vieille Juive, consciente qu' on parlait d'elle, baissait les yeux dans son sac. Tout le long de la rue Sainte-Catherine ouest, les nez se collaient aux vitres en hiver, les bras s'appuyaient sur le rebord des fenêtres en été. [...] Les dernières descendaient chez Eaton, au coin d'University. Jamais personne du groupe n' allait plus loin que chez Eaton. À l'ouest de ce grand magasin c'était le grand inconnu: l'anglais, l'argent, Simpson's, Ogilvy's, la rue Peel, la rue Guy, jusqu' après Atwater, là où l'on recommençait d̀ se sentir chez soi à cause du quartier Saint-Henri, tout proche et de l'odeur du port. 
Mais jamais personne n' allait jusqu'd Saint-Henri et jamais personne de Saint-Henri ne venait jusqu' au Plateau Mont-Royal. (GF, p. 22-25)

Plus que le tracé poursuivi par le tramway, cette scène est fort éclairante sur les limites intériorisées (une forme d'autocensure presque) du quartier-ghetto, parce que les femmes du Plateau ne font précisément que passer par la rue Saint-Laurent, elles n'y descendent pas, et l'on voit très bien comment elles portent inscrites en elles, jusque dans leurs propos et leur langue même, les marques du quartier auquel elles appartiennent. Malgré l'atmosphère joyeuse, euphorique, quelque peu hystérique même de la scène, les désagréables remarques racistes à l'endroit de Sam Katz ${ }^{23}$ et de la vieille Juive disent très bien le rejet de toute altérité, de toute différence, et scellent le prix à payer pour assurer la cohésion du clan. D'ailleurs, on sent bien que ce n'est que parce qu'elles sont ici ensemble dans un lieu protégé, le tram, qu'elles se permettent ces agressions pour rire. Les femmes du Plateau de Tremblay peuvent circuler hors du quartier, mais selon un trajet déterminé, dont le but est fixé; elles restent passives, elles n'entrent pas en contact avec l'autre, elles ne transgressent pas les limites du périmètre sacré et rentrent intactes à la maison.

La scène se déroule tout autrement dans Maryse où tout est moins affaire de provisions et d'exotisme à bon compte qu'histoire de langues et d'identité; la scène est d'ailleurs enlevée sous le mode d'une joute oratoire au moment où Maryse, Miguel (un Chilien) et MLF se retrouvent, eux, à l'intérieur du magasin d'alimentation de la rue Saint-Laurent:

Ils firent les boutiques de la rue Saint-Laurent et atterrirent finalement chez Warshaw ou Miguel, presque rendu d la caisse, commença une belle chicane en espagnol avec un Portugais pendant que MarieLyre s'engueulait en anglais avec une Grecque complètement dépassée par l'énergie furieuse qui émanait de ce couple peu banal mais si bien assorti. La discussion avait pour thème la conduite des chariots colossaux dans les allées encombrées, étroites et sinueuses du magasin d'alimentation Warshaw. MLF et Miguel prirent à partie tout le monde, dans toutes les langues qu'il connaissaient deux deux mais en se gardant bien d' utiliser le français: c'eût été trop facile! La dispute allait dégénérer en bagarre générale quand $M L F$ perçut le danger: pendant l'altercation, une Québécoise (qui était en fait Canadienne mais ça ne se voyait pas du tout), un Juif, deux Portugaises et une

23 En dépit des coups de griffe qui durcissent le nom par des lettres bizarres $(k, z)$, $K a t z$ s'inscrit bien entendu dans la lignée de ces chats étranges et familiers, très Unheimleich qui, du Matou à Duplessis et à Mélibée Marcotte, sans oublier la fidèle Auchimine tuée par une machine de la dédicace de l'Hiver de force, font partie de ces chroniques et se présentent souvent, dans ces différents univers romanesques, comme des symptômes d'altérité, le masque agressif ou amical, déraisonnable et fantastique de la vie inconsciente, toujours prête à faire imuption. Comme le rappelle Ducharme dans l'une de ses exergues: $L$ 'homme est le meilleur ami du chat, c'est pourquoi il faut y penser deux fois avant de faire des affaires pour le faire disparaitre. 
famille entière d'Italiens s'étaient faufilés devant eux da la caisse. $O$ rage! Sur l'autre front, la situation n'était guère plus reluisante: le gérant de l'endroit ramait péniblement vers eux. Or Marie-Lyre, en bonne ménagère, avait glissé dans son sac d main un Bourseault bien mûr, qu' elle ne payait jamais, vu la cherté de ces petites choses. Elle jugea qu'ils s'étaient suffisamment fait remarquer, mit ses doigts dans sa bouche et siffla comme son frère Clément le lui avait appris vingt ans plus tôt. Aussitôt, les principaux protagonistes de la joute oratoire, c'est-à-dire la Grecque et le Portugais sus-mentionnés, de même que Miguel et elle-même, se ressaisirent, se serrèrent la main et mirent le cap vers leurs caisses respectives.

- Maudits émigrés, dit une cliente, c'est des vrais sauvages!

Elle dardait un oeil noir sur MLF qui déclara très haut, avec un accent fortement gaspésien:

- $J$ ' te l'avais dit, itou, mon pitou, que j'avais pas d'l'air pantoute d'une Pellequier du Bas du fleuve (sa mère était une PelletierBarlette) mais d' une wops à grand yeulle.

Elle jubilait. Ils laissèrent la Québécoise pantoise, doutant presque de ses propres origines l'abord-d-plouffiennes, et sortirent en distribuant de larges sourires à leur auditoire. (M, p. 381-382)

Cette bataille de langues - véritable petite Babel de la confusion: et n'oublions pas que par Babel, Dieu déclara la guerre et rendit désormais impossible toute adéquation entre la langue et l'identité du sujet comme une et indivisible - prend ici la forme éminemment comique (on n'est pas loin de la bataille des timbres des Belles-Sœurs, et pourtant on est aussi à des annéeslumière de cette scène-cuisine) d'une formidable engueulade chez Warshaw (la guerre se lit d'ailleurs jusque dans le nom du magasin). Cette petite scène dit assez clairement ce qui s'est déplacé dans la question de l'identité, avec cette nouvelle et précaire alliance stratégique avec les autres cultures. Remarquons au passage que MLF ne peut s'empêcher de piquer un Boursault ${ }^{24}$, ce qui n'est pas rien tout de même, petit symbole hors de prix - vu la cherté de ces petites choses - de la France. Un certain rapport difficile à la culture nourricière est encore sensible dans ce petit geste, où l'ọn dérobe un petit quelque chose à la mère patrie, sans vouloir en payer le prix, et ce n'est certes pas un hasard si la jubilation de cette scène passe toute par l'oralité, langue et nourriture confondues. Dans l'univers de Maryse, il n'y a plus supériorité d'une culture sur une autre, il n'y a plus revendication d'une identité et d'origines pure laine comme celle de cette Québécoise dépassée par la situation et doutant presque de ses propres origines l'abord- $d$-plouffiennes (et dans ce signifiant, c'est l'image mythique de la famille québécoise par excellence, les Plouffe, qui résonne à

24 Bourseault: la coquille souligne la portée du geste manqué. Dérober même une de ces petites choses n'est pas ici innocent, une lettre en trop vient encore signaler le larcin. 
notre oreille et est du coup ridiculisée), il n'y a plus qu'une joyeuse mêlée où les contradictions, la passion [...] pour les débats contradictoires publics et improvisés (M, p. 382), sont avivées pour la plaisir. Cette transculture heureuse, ludique, appelant une autre circulation de la langue et de l'identité, est-elle le nouveau mythe, l'utopie sociale du Québec des années quatre-vingt? Il y aurait peut-être lieu, pour rompre l'oscillation un peu trop symétrique qui risque de s'installer à la lecture de ces deux scènes, d'intercaler une troisième prise de la Main, petite scène tirée de l'Hiver de force, cette autre chronique du Plateau Mont-Royal dont je n'ai pu parler ici et qui s'inscrit chronologiquement entre celles de Tremblay et de Noèl. La scène se passe au moment où les Ferron et Laïnou (qui habite sur l'avenue Draper, c'est-à-dire le quartier bourgeois N.D.G.) sortent du restaurant Moishe où elle les a invités et où ils ont bu quatre bouteilles de Chateauneuf-du-pape:

Juste en face de chez Moishe se dressent, sales, gris, pleins de carreaux brisés, ces espèces de camps de concentration de couture où des Grecques, des Espagnoles, des Italiennes, toutes noires, comme brûlées jusqu' au charbon, importées pour les mêmes raisons que les tissus qu'elles façonnent, peinent tous les jours pour gagner en une semaine ce qu' on venait de manger tristement en une heure. On a regardé ostensiblement les façades macabres, on a pris un air dégoûté et on a demandé à Laïnou si elle n'avait pas honte.

- C'est elles qui devraient avoir honte! C'est pas un exemple à donner à leurs enfants! C'est d cause des connes comme elles que des conneries comme ça peuvent se perpétuer! Moi je les ferais toutes coffrer! En prison tous les soumis! Moi je laisserais en liberté rien que les gens comme moi, qui aimeraient mieux mourir que se faire traiter comme ça! À la génération suivante, plus de problèmes, tout le monde irait manger chez Moishe quand ça lui tenterait!

On boude. Il y a des bornes au-dela desquelles l'indigence intellectuelle arrogante d' une grande amie fait mal, blesse, déçoit, choque. ${ }^{25}$

Plus que les propos intolérablement racistes de Laïnou ${ }^{26}$, durcissement de la honte qui tremblait déjà dans le rire hystérique des femmes du tram chez Tremblay, lorsqu'elles cherchaient à exclure l'autre par leurs quolibets, en le plaçant à distance, sous le signe de l'étrangeté menaçante, plus que la solidarité spontanée des Ferron avec toutes leurs minorités, adhésion qui anticipe ce qui passe, à la génération suivante très précisément, dans la chronique de Francine Noël où l'altérité euphorique et fusionnelle est exaltée à tout prix, fût-ce à celui de s'interdire, même par jeu, parce que c'eût été trop facile, sa propre langue, ce qui frappe ici c'est la représentation d'une réalité le plus souvent occultée de

25 Réjean Ducharme, l'Hiver de force, op. cit., p. 209-210.

26 Il faut même prêter l'oreille à la montée irrésistible du «Moi» dans cette tirade de Laînou où l'affirmation de soi écrase tout autour d'elle, ce qui provoque le retrait des Ferron, l'effacement de leur sujet: On boude. 
Montréal, ces espèces de camps de concentration de couture, ces façades macabres aux carreaux brisés d'entrepôts, qui seront convertis à la génération suivante en lofts occupés par des gens comme Laïnou. Chez Ducharme, l'affect lié à cette prise de conscience est fait de honte comme chez Tremblay, mais pas pour les mêmes raisons; la frontière ne passe plus entre les Québécois et les autres (Italiens, Portugais, Grecs, etc.), mais entre les Québécois eux-mêmes, comme chez Noël: l'identité, tout comme la perception de la ville, est loin ici d'être une et indivisible.

Car en matière d'identité (et non pas d'identification), les choses restent toujours très mouvantes, indéfinies et ouvertes. Comme l'écrit Ducharme à propos des bornes qu'il vaut mieux savoir franchir avec discemement, c'est aussi toujours aux frontières, aux zones d'intersections communes, qu'on sent commencer à bouger ce qui se répercutera demain à l'intérieur du quartier et métamorphosera soudain la forme même de la ville.

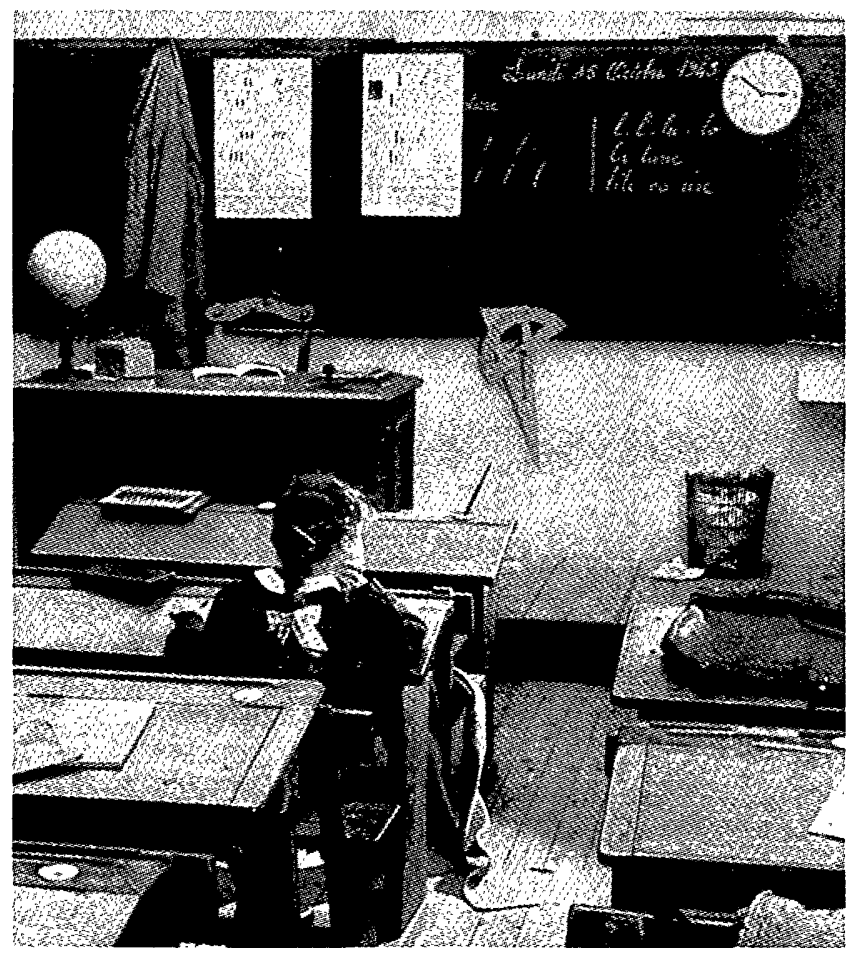

Valérie Clément 